(C) 2017 Optical Society of America. One print or electronic copy may be made for personal use only. Systematic reproduction and distribution, duplication of any material in this paper for a fee or for commercial purposes, or modifications of the content of this paper are prohibited.

\title{
Design rules for combined label-free and fluorescence Bloch surface wave biosensors
}

\author{
Francesco Michelotti, ${ }^{1,}{ }^{*}$ Riccardo Rizzo, ${ }^{1,2}$ Alberto Sinibaldi, ${ }^{1}$ Peter \\ Munzert, ${ }^{2}$ Christoph WÄChter, ${ }^{2}$ AND NORbert DANZ ${ }^{2}$ \\ ${ }_{1}^{1}$ Dipartimento di Scienze di Base e Applicate per I'Ingegneria, SAPIENZA Università di Roma, Via A. Scarpa 16, 00161 Roma, Italy \\ 2 Fraunhofer Institute for Applied Optics and Engineering, Albert Einstein Strasse 7, 07745, Jena, Germany \\ *Corresponding author: francesco.michelotti@uniroma1.it
}

Received XX Month XXXX; revised XX Month, XXXX; accepted XX Month XXXX; posted XX Month XXXX (Doc. ID XXXXX); published XX Month XXXX

\begin{abstract}
We report on the fabrication and physical characterization of optical biosensors implementing simultaneous label-free and fluorescence detection and taking advantage of the excitation of Bloch surface waves at a photonic crystal's truncation interface. Two types of purposely-designed one-dimensional photonic crystals on molded organic substrates with micro-optics were fabricated. These feature either high or low finesse of the Bloch surface wave resonances and were tested on the same optical readout system. The experimental results show that designing biochips with a large resonance quality factor does not necessarily lead in the real case to an improvement of the biosensor performance. Conditions for optimal biochips' design and operation of the complete bio-sensing platform are established. (C) 2017 Optical Society of America
\end{abstract}

OCIS codes: (280.4788) Optical sensing and sensors; (280.1415) Biological sensing and sensors (240.6690) Surface waves; (230.5298) Photonic crystals.

http://dx.doi.org/10.1364/XXXXXXX

Optical sensors based on the resonant excitation of electromagnetic surface waves are widely diffused in the field of biochemical binding analysis. In particular, surface plasmon resonance (SPR) is involved in many label-free sensing platforms, due to the sensitivity, resolution and simplicity of the detection scheme [1]. Recently, Bloch surface waves (BSW) [2] sustained at the interface between a finite one-dimensional photonic crystal (1D-PC) and an external medium were proposed as an alternative to SPR $[3,4]$. They feature an improved limit of detection [5] and the possibility to operate in a wide spectral range from the visible to the Terahertz region, when properly selecting the $1 \mathrm{D}-\mathrm{PC}$ materials and geometry [6-7]. Coupling to BSW has also been demonstrated to improve excitation and collection of the fluorescence emitted by molecules at the surface of a low loss dielectric 1D-PC [8].
Recently we reported on the development of sensing platforms implementing simultaneous label-free (LF) and fluorescence (FLUO) detection, based on BSW and operating in either the near infrared [9] or visible [10] ranges. Such an approach aims at increasing the resolution by simultaneously collecting complementary signals, similar to other configurations [11-12].

In this Letter we report on the design, fabrication and experimental characterization of BSW sensors featuring two different $1 \mathrm{D}-\mathrm{PC}$ designs. The aim is to identify the route to optimized sensors integrating both LF and FLUO operation in the visible range [10], respectively at $\lambda_{\mathrm{LF}}=670 \mathrm{~nm}$ and $\lambda_{\text {FLUO }} \in[670$ $\mathrm{nm}, 750 \mathrm{~nm}$ ] for photo-excitation carried out at $\lambda_{\mathrm{Exc}}=635 \mathrm{~nm}$.

The 1D-PC used in the experiments are dielectric multilayers deposited on cyclic olefin copolymer (TOPAS) substrates [13-14] by plasma-ion-assisted deposition (PIAD) under high vacuum [15].

TABLE I. Geometry of the Type 1 and 2 sensors

\begin{tabular}{|c|c|c|c|c|c|}
\hline Type & Geometry & $\mathrm{N}$ & & & $\begin{array}{l}\text { Topping } \\
\text { bi-layer }\end{array}$ \\
\hline & & & & $\mathrm{d}_{\mathrm{L}}$ & $\mathrm{dH}_{\lfloor\mathrm{nm}\rfloor}^{\mathrm{dL}_{\mathrm{L}}{ }^{\prime}}$ \\
\hline 1 & \multirow{2}{*}{$\mathrm{L}(\mathrm{HL})^{\mathrm{N}} \mathrm{H}^{\prime} \mathrm{L}^{\prime}$} & 3 & 120 & 340 & $\begin{array}{c}\mathrm{Ta}_{2} \mathrm{O}_{5} \\
20\end{array}$ \\
\hline 2 & & 2 & 120 & 275 & $\begin{array}{c}\mathrm{TiO}_{2} \\
20\end{array}$ \\
\hline
\end{tabular}

The geometries of the two sensor types (Table I) share a common periodic multilayer and are terminated by two different thin dielectric bi-layers. $\mathrm{SiO}_{2}$ and $\mathrm{Ta}_{2} \mathrm{O}_{5}$ are the low and high index dielectric materials used to fabricate the periodic part, with $\mathrm{N}$ periods; the complex refractive indices are $\mathrm{n}_{\mathrm{L}}=1.474+\mathrm{i} 5^{*} 10^{-6}$ and $\mathrm{n}_{\mathrm{H}}=2.106+\mathrm{i} 5^{*} 10^{-5}$ at $\lambda_{\mathrm{LF}}$, respectively. The thin dielectric bi-layer is either $\mathrm{Ta}_{2} \mathrm{O}_{5} / \mathrm{SiO}_{2}$ or $\mathrm{TiO}_{2} / \mathrm{SiO}_{2}$, for Type 1 and 2 sensors respectively. $\mathrm{TiO}_{2}$ has a complex refractive index $\mathrm{n}_{H^{\prime}}=2.28+\mathrm{i} 1.8^{*} 10^{-3}$ at $\lambda_{\mathrm{LF}}$. In the topping bi-layer, the role of the first high index layer $\left(\mathrm{Ta}_{2} \mathrm{O}_{5}\right.$ or $\left.\mathrm{TiO}_{2}\right)$ is to tailor the $\mathrm{BSW}$ properties to improve the LF biosensing performance, while the second layer $\left(\mathrm{SiO}_{2}\right)$ provides a surface that can be treated with standardized and reliable silane based chemical functionalization protocols [10]. 
The TOPAS substrates $\left(\mathrm{n}_{\mathrm{TOPAS}}=1.530\right.$ at $\left.\lambda_{\mathrm{LF}}\right)$ were fabricated by injection molding and include two cylindrically shaped input and output optical windows as shown in Fig. 1. The substrates fit to the optical reading system of an integrated platform designed and fabricated to operate in both LF and FLUO modes. The illumination is focused cylindrically along the incidence plane to a line at the surface of the chip. The output cylindrical window of the chip (output diopter) is used to collect the reflected/emitted light and performs optical Fourier transformation at the CCD (Sony ICX205AL) array sensor along the incidence plane direction.

As shown in Fig. 1(a), in the LF mode a collimated laser beam at $\lambda_{\mathrm{LF}}$ is focused at the chip surface above the total internal reflection (TIR) angle and the reflected beam is collected by the CCD array in a 4 deg angular window. Destruction of the spatial coherence by means of a rotating diffuser (not shown), combined with the CCD integration time, avoids image degradation due to speckles. In such a Kretschmann-Raether configuration [16], the excitation of a BSW gives rise to a dip in the angular reflectance spectrum that is transformed by the output diopter to a dip in the intensity distribution along the CCD long axis (1280 pixel, in the incidence plane).
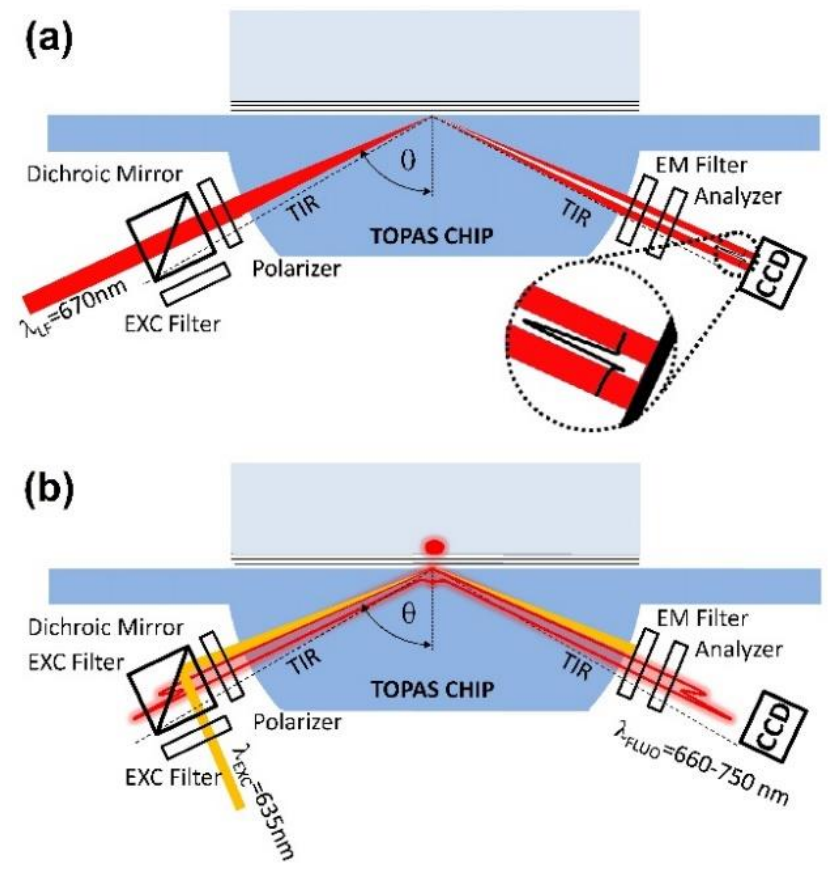

Fig. 1. Sketch of the optical configuration of the sensing platform for the (a) LF operation, and the (b) FLUO operation. For the sake of clarity, in the sketch we did not draw the beam shaping optics and beam steering mechanics. Dimensions are not to scale.

In the FLUO mode, see Fig.1(b), we use a BSW for both fluorescence excitation and collection. A collimated laser beam at $\lambda_{\mathrm{EXC}}$ is focused in a narrower angular range $\left(\Delta \theta_{\mathrm{ExC}} \sim 2 \mathrm{deg}\right) . \mathrm{A}$ combination of a dichroic mirror and excitation and emission filters (Chroma, F46-112 633-640 nm ET Laser Filter Set) limits stray light effects. Parallel steering in the incidence plane of the fluorescence excitation beam (not shown in Fig.1) allows one to tune the incidence angle $\theta_{\mathrm{Exc}}$ to match the BSW angle $\theta_{\mathrm{BSW}}$ at $\lambda_{\mathrm{EXC}}$, and excite fluorophores placed at the chip surface via a resonant BSW mode. Previous works [8] demonstrated that the field localization due to the excitation of a BSW gives rise to a strong increase of the excitation rate. Moreover, the broad emission spectrum of the fluorophores is coupled to polychromatic BSWs, which leak in-to the substrate in a narrow angular range [17], and are collected by the same CCD. The output diopter, combined with the disper-sion of the BSW, images every spectral component of the emission with a one-to-one mapping onto a different position of the $\operatorname{CCD}[9,10]$.

In both the LF and the FLUO operation, the intensity along the CCD short axis (1024 pixel) corresponds to different positions along the illuminated strip at the chip surface, allowing sensing several spots at the same time [14]. A polarizer can set the linear polarization of the incident light and an analyzer, as shown in Fig. 1 , analyzes that of the reflected/emitted light.

The 1D-PC geometries resumed in Table I were designed to show either a narrow or a broad BSW dip in the LF angular reflectance spectrum $\mathrm{R}_{\mathrm{TE}}$ for the TE polarization. In Fig. 2(a) and Fig. 3(a) we show the theoretical $\mathrm{R}_{\mathrm{TE}}$ at $\lambda_{\mathrm{LF}}$ for the two sensor types, calculated [16] assuming a TOPAS substrate $\left(\mathrm{n}_{\text {TOPAS }}=1.530\right)$ and water as external medium ( $\left.\mathrm{n}_{\mathrm{EXT}}=1.330\right)$. For both types, deep resonances at $\theta_{\mathrm{BSW}}$ are expected for the TE polarized BSW, which are strongly localized at the 1D-PC sensor surface. The sensors also feature shallow and broad TM resonances, corresponding to modes that are more extended inside the 1D-PC structure.

In Table II we list the values of the full width half-maximum (W) and resonance depth (D) for all dips appearing in Fig. 2(a) and Fig.3(a), together with their calculated volume sensitivity $\mathrm{S}_{\mathrm{LF}}=\mathrm{d} \theta_{\mathrm{BSW}} / \mathrm{dn}_{\mathrm{EXT}}$. From the values found for $\mathrm{W}, \mathrm{D}$, and $\mathrm{S}_{\mathrm{LF}}$ one can evaluate the figure of merit for the LF performance defined as $\mathrm{FoM}_{\mathrm{LF}}=\mathrm{S}^{*} \mathrm{D} / \mathrm{W}$ [16], which is listed in Table II too. We also list the calculated field enhancement factor (FEF), defined as the ratio of the amplitude of the field at the sensor/water interface to that of the field in TOPAS, when the chips are excited at $\lambda_{\mathrm{EXC}}$ at the TE $\theta_{\mathrm{BSW}}$.

Among all cases, the Type 1 sensor displays the best design FoM $_{\mathrm{LF}}$ and FEF (TE pol), that could suggest it as a first choice for both label-free and fluorescence biosensing applications. However, as it will be clear from the experimental results reported below, the best choice reveals to be instead that of Type 2 sensor.

In Fig. 2(b) and Fig. 3(b), we show the angular LF reflectance measured experimentally when operating the chips on the platform of Fig. 1, for the Type 1 and 2 respectively. Data were obtained by averaging 30 CCD lines, corresponding to a $260 \mu \mathrm{m}$ wide spot on the chip surface (also for FLUO operation,).

In the TE case (red), sharp resonant dips are found. As expected, the resonant TM dips are hardly observed (not shown); however, their angular position can be probed by operating the platform in a phase-sensitive configuration (CROSS), where the two polarizers are turned by $45 \mathrm{deg}$ with respect to the incidence plane. Under such conditions, the overall reflectance $R_{\text {CROSS }}$ is sensitive to the amplitude and phase of the TE and TM reflected components [16]. In Fig. 2(b) and Fig. 3(b), the experimental R $\mathrm{R}_{\text {CRoss (black) shows }}$ resonances around the expected position of the TM modes and permits to identify them.

Besides allowing for modes spectroscopy and characterization, working in the CROSS configuration is very desirable for sensing applications as it permits increasing the FoM when the 1D-PC are characterized by extremely small losses (and small D) [16]. For such a reason polarizers were implemented in the integrated optical platform. We point out that in the CROSS case the $S_{L F}$ coincides with that of either the TE or the TM single polarization case, depending on the resonance that is exploited [16]. 


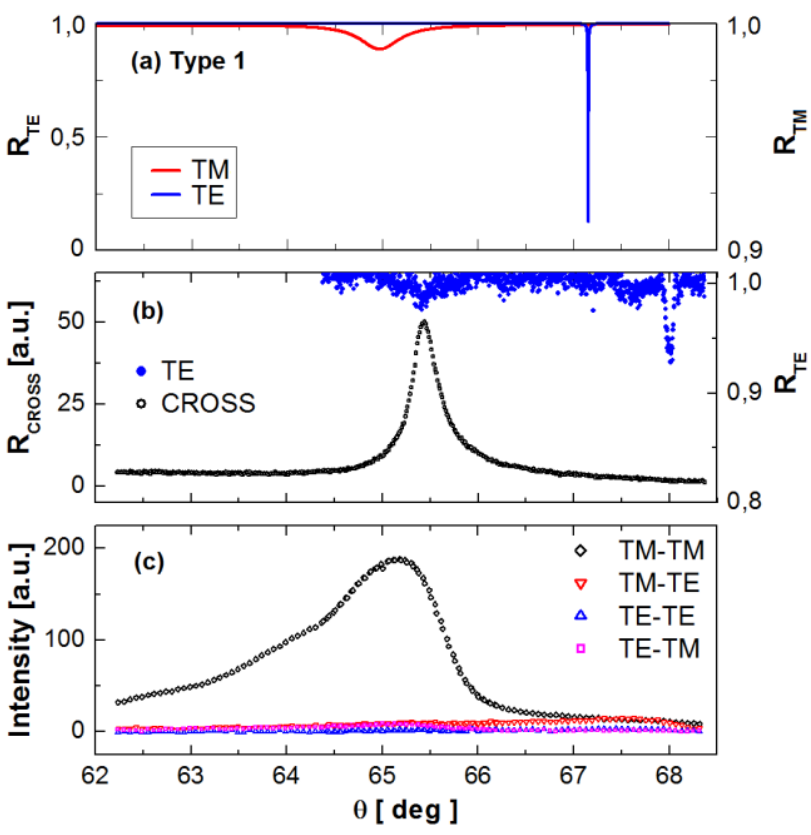

Fig. 2. Type 1. (a) Simulated angular TIR reflectance, (b) Experimental reflectance in the TE and CROSS configuration, (c) Fluorescence emission for four different excitation (1 $1^{\text {st }}$ label) and collection $\left(2^{\text {nd }}\right.$ label) polarizations.

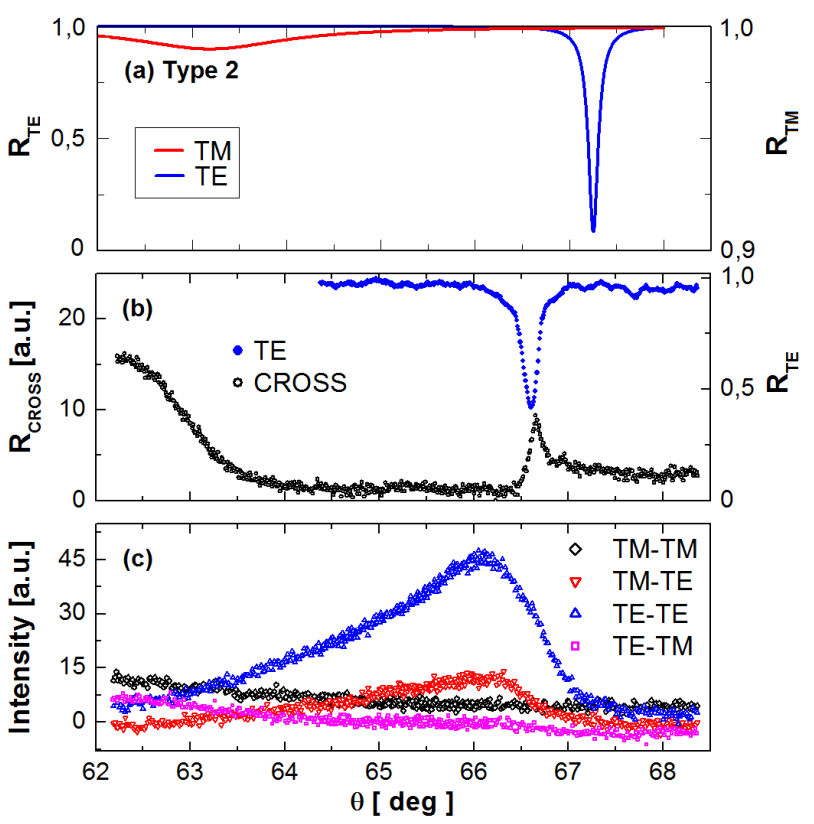

Fig. 3. Type 2. (a) Simulated angular TIR reflectance, (b) Experimental reflectance in the TE and CROSS configuration, (c) Fluorescence emission for four different excitation ( $1^{\text {st }}$ label) and collection $\left(2^{\text {nd }}\right.$ label) polarizations.

TABLE II - Theoretical and experimental values of the relevant parameters for the two 1D-PC designs.

\begin{tabular}{|c|c|c|c|c|c|c|c|c|c|c|}
\hline \multirow[b]{3}{*}{ Type } & \multirow[b]{3}{*}{ Pol } & \multicolumn{5}{|c|}{ THEORY } & \multirow{2}{*}{\multicolumn{4}{|c|}{$\begin{array}{c}\text { EXPERIMENT } \\
\lambda_{\mathrm{LF}} \\
\end{array}$}} \\
\hline & & \multicolumn{4}{|c|}{$\lambda_{\mathrm{LF}}$} & \multirow{2}{*}{$\frac{\lambda_{\mathrm{EXC}}}{\mathrm{FEF}}$} & & & & \\
\hline & & $\begin{array}{c}\text { W } \\
{[\text { mdeg }]}\end{array}$ & $\mathrm{D}$ & $\begin{array}{c}\mathrm{S}_{\mathrm{LF}} \\
{[\mathrm{deg} / \mathrm{RIU}]}\end{array}$ & $\begin{array}{c}\mathrm{FoM}_{\mathrm{LF}} \\
{\left[\mathrm{RIU}^{-1}\right]}\end{array}$ & & $\begin{array}{c}\mathrm{W} \\
{[\mathrm{mdeg}]}\end{array}$ & $\mathrm{D}$ & $\begin{array}{c}\mathrm{S}_{\mathrm{LF}} \\
{[\mathrm{deg} / \mathrm{RIU}]}\end{array}$ & $\begin{array}{c}\mathrm{FoM}_{\mathrm{LF}} \\
{\left[\mathrm{RIU}^{-1}\right]}\end{array}$ \\
\hline \multirow{2}{*}{1} & $\mathrm{TE}$ & 6 & 0.85 & 29.4 & 4165 & 64 & 60 & 0.08 & 26.7 & 35.6 \\
\hline & $\mathrm{TM}$ & 550 & 0.01 & 22.7 & 0.41 & 8 & NA & NA & 21.3 & NA \\
\hline \multirow{2}{*}{2} & $\mathrm{TE}$ & 140 & 0.90 & 35.4 & 227 & 16 & 200 & 0.55 & 31.8 & 87.5 \\
\hline & TM & 2090 & 0.01 & 33.7 & 0.16 & 4 & NA & NA & $\mathrm{NA}$ & NA \\
\hline SPR & $\mathrm{TM}$ & \multicolumn{5}{|c|}{ After Ref. $5, \lambda_{\mathrm{LF}}=804 \mathrm{~nm}$} & 1470 & 0.76 & 80.1 & 41.4 \\
\hline
\end{tabular}

For both sensor types, the BSW resonances are slightly shifted with respect to the theoretical position. For Type 1 , the TE dip is much wider and shallower than the theoretical prediction, while for the Type 2 the dip, which is wider by design, is not much broadened and keeps relatively deep. Such effects are ascribed, for both Type 1 and 2, to 1DPC layers' thickness fabrication tolerances [18] and to the plastic substrate optical quality [5]. Additionally, for Type 2, the $\mathrm{TiO}_{2}$ absorption loss might differ from that assumed for the simulations.

Reference measurements carried out with the same 1D-PC deposited on microscope slides show that the higher optical quality of the substrates preserves the dips' W (not reported here).

From the LF measurements, we extracted the experimental values for $\mathrm{W}, \mathrm{D}$ and $\mathrm{S}_{\mathrm{LF}}$ shown in Table II. The $\mathrm{S}_{\mathrm{LF}}$ values were measured experimentally by using different known solutions of glucose in doubly deionized water [5]; in the TM BSW case (Type 1 only) $S_{L F}$ was measured in the CROSS configuration. In Table II, the experimental values for the FoM are significantly reduced compared to theoretical predictions and indicate that the Type 2 sen- sor shows the best LF performance. For comparison we report also the $\mathrm{FoM}_{\mathrm{LF}}$ for a SPR sensor operating at $\lambda_{\mathrm{LF}}=804 \mathrm{~nm}$ [5], showing that indeed the present $1 \mathrm{D}-\mathrm{PC}$ provides improved LF performance.

Fig. 2(b) and Fig. 3(b) also show that the CROSS operation enhances the TE and TM response of the Type 2 sensors, whereas for the Type 1 the TM resonance is enhanced but the TE is depressed. Such last result is due to a reduced phase shift $(<2 \pi)$ of the TE reflection coefficient across such a high finesse resonance [16].

In Fig. 2(c) and Fig. 3(c), we show measurements carried out in the FLUO mode in water environment. Before carrying on the measurements, the surface of the sensors was stained with a $10 \mathrm{nM}$ solution of Nile Blue (NB) dye (Radiant Dyes, $\lambda_{\operatorname{MAX}}=685 \mathrm{~nm}$ ) [19] in deionized water and rinsed repetitively. The gain and integration time of the CCD were increased with respect to the LF case.

The fluorescence emission curves were obtained by exciting at $\lambda_{\text {ExC }}$ with either TE or TM polarization and detecting fluorescence along either the TE or the TM polarization. Depending on the polarization, the excitation angle $\theta_{\mathrm{Exc}}$ was tuned to the respective 
resonance by maximizing the fluorescence signal, if present. As the $\mathrm{NB}$ molecules are free to rotate, their orientation is lost due to rotational randomization in the NB excited state [20]; therefore NB molecules can be assumed to be isotropically oriented and couple to both TE and TM polarized BSW of the 1D-PC.

For the Type 1 (Fig. 2(c)), relatively intense fluorescence was measured only for the TM-TM case. Owing to the dispersion of the TM BSW mode, each spectral component of the emission at $\lambda>$

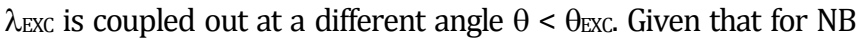
$\lambda_{\mathrm{MAX}}>\lambda_{\mathrm{LF}}>\lambda_{\mathrm{EXC}}$ the angular fluorescence distribution lies on the small angles side of the resonance observed in the LF mode at $\lambda_{\mathrm{LF}}$. The NB fluorescence coupled to the TM BSW is channeled in a 4 deg angular window and can be efficiently detected by the same optical system used for LF operation. Only a faint TM-TE fluorescence signal is observed, on the small angle side of the TE resonance in the LF mode, despites the extremely large theoretical FEF of the TE mode. TE excitation does not give rise to any appreciable fluorescence signal.

For the Type 2 (Fig. 3(c)), relatively intense fluorescence was measured for all TM-TE combinations, with angular distribution that lies on the small angles side of the respective LF resonance. The TM fluorescence however peaks outside the observation window at smaller angles. As expected from the FEF values, the TE emitted fluorescence is larger for TE than for TM excitation. The intensities found in Fig. 3(c) and Fig. 2(c) cannot however be compared, because the NB staining procedure suffers from poor reproducibility and does not permit to control the NB surface density.

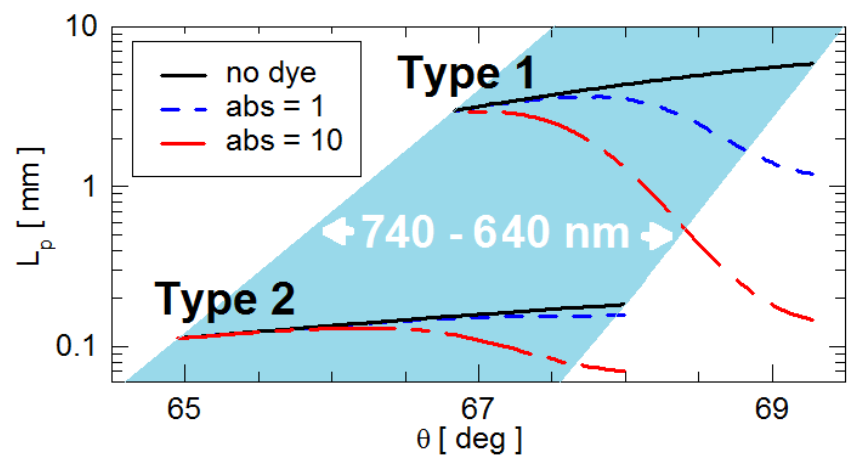

Fig. 4. Calculated $L_{p}$ for both Type 1 and 2 design sensors. No dye and no absorption losses (solid), arbitrary 1-fold (dashed) and 10-fold (dotdashed) NB dye absorption at the 1D-PC surface. The light blue region is just a guide for the eye, suggesting that the same spectral emission range for the NB dye corresponds to different angular ranges for the two types, due to the different BSW dispersion.

The completely different fluorescence characteristics observed for the two types of sensors can be ascribed to the following causes.

First of all, the NB dye excitation rate depends on the FEF of the BSW at $\lambda_{\text {Exc }}$ and on the ratio $\eta=W / \Delta \theta_{\text {Exc }}$. For the Type 1 sensor $\eta \sim 3 \%$ for the TE polarization and, despites the largest FEF, the excitation rate is small. On the other hand, for the Type 1 (TM pol) and for the Type $2, \eta$ is larger and consequently the excitation rate is improved.

Second, the propagation length $\mathrm{L}_{p}$ of the TE BSW mode, excited by spontaneous emission, before radiating in the substrate is extremely large in Type 1 sensors. In Fig. 4, we show the calculation of $\mathrm{L}_{p}$ in the TE case, carried out with a commercial mode solver for the two sensor types (the TM values are more than 10 times smaller). We performed the calculations for several different arbitrary values of the NB dye absorption. As the effect of increasing NB dye absorption losses at the 1D-PC surface shown in Fig. 4 illustrates, the increased $\mathrm{L}_{p}$ also sensitizes the stack for reabsorption. As a consequence, for the Type 1 (TE pol) $\mathrm{Lp}_{\mathrm{p}}$ is so large that the emitted energy is strongly reabsorbed by the NB dye and the remaining energy leaves the 1D-PC outside the spatial field of view of the detection optics, which is about $2 \mathrm{~mm}$.

As a conclusion we can state that designing 1D-PC with extremely sharp resonances does not necessarily lead to improved LF and FLUO sensors. For LF sensing the design must carefully take into account the fabrication tolerances and the substrate quality that limit the FoM $\mathrm{MF}_{\mathrm{LF}}$. For FLUO applications, the sharpness of a resonance converts into a large propagation length, which governs both the angular matching of the excitation beam to the in-coupling BSW and the extraction of the out-coupling BSW in terms of real-space widths of the collecting system. This must be kept in mind also for applications in fluorescence microscopy. The Type 2 design allows for a FoM $\mathrm{LF}_{\mathrm{LF}}$ which outperforms SPR and at the same time permits to operate efficiently in fluorescence mode.

Funding. This work was funded by the European Commission through the project BILOBA (Grant Agreement 318035).

\section{References}

1. M. Piliarik and J. Homola, Opt. Expr., 17, 16505 (2009).

2. P. Yeh, A. Yariv, C. -S. Hong, J. Opt. Soc. Am. 67, 423 (1977).

3. M. Shinn, W. M. Robertson, Sensors and Actuators B 105, 360 (2005).

4. V. N. Konopsky and E. V. Alieva, Anal. Chem. 79, 4729 (2007).

5. A. Sinibaldi, N. Danz, E. Descrovi, P. Munzert, U. Schulz, F. Sonntag, L. Dominici, and F. Michelotti, Sens. Act. B 174, 292 (2012).

6. A. Farmer, A. Friedli, S. M. Wright, W. M. Robertson, Sens. Act. B 173, 79 (2012).

7. V. Paeder, J. Darmo, K. Unterrainer, IEEE 38th International Conference on Infrared, Millimeter, and Terahertz Waves (IRMMW-THz), DOI: 10.1109/IRMMW-THz.2013.6665717 (2013).

8. J. Y. Ye, M.Ishikawa, Opt. Lett. 33(5), 1729 (2008).

9. A. Sinibaldi, A. Fieramosca, R. Rizzo, A. Anopchenko, N. Danz, P. Munzert, C. Magistris, C. Barolo, F. Michelotti, Opt. Lett. 39, 2947 (2014).

10. A. Sinibaldi, C. Sampaoli, N. Danz, P. Munzert, L. Sibilio, F. Sonntag, A.Occhicone, E. Falvo, E. Tremante, P. Giacomini, F. Michelotti, Biosens. Bioelectron. 92, 125 (2017)

11. C. Mathias, N. Ganesh, L.L. Chan, B.T. Cunningham, Appl. Opt. 46(12), 2351 (2007).

12. K. Toma, M. Vala, P. Adam, J. Homola, W. Knoll, J. Dostálek, Opt. Expr., 21(8), 10121 (2013).

13. http://www.topas.com/sites/default/files/files/TOPAS_Brochure_E_201 4_06\%281\%29.pdf.

14. N. Danz, A. Kick, F. Sonntag, S. Schmieder, B. Höfer, U. Klotzbach, M. Mertig, Eng. Life Sci. 11, 566 (2011).

15. P. Munzert, N. Danz, A. Sinibaldi, F. Michelotti, Surf. Coat. Tech., 314, 79 (2017).

16. A. Sinibaldi, R. Rizzo, G. Figliozzi, E. Descrovi, N. Danz, P. Munzert, A. Anopchenko, and F. Michelotti, Opt. Expr., 2, 23331 (2013).

17. R. Badugu, K. Nowazcyk, E. Descrovi and J. R. Lakowicz, Anal. Biochem. 442 (1), 83 (2013).

18. R. C. Nesnidal, T.G. Walker, Appl. Opt. 35(13), 2226 (1996).

19. http://www.radiant-dyes.com/index.php, Nilblau perchlorate (Cat.N.98). 20. F. Perrin, J. Physique 7, 390 (1926). 